\title{
SELECTIVE NECK DISSECTION IN NODE POSITIVE SQUAMOUS CELL CARCINOMA OF HEAD AND NECK
}

\author{
Babak Givi, MD', Gary Linkov, BS ${ }^{2}$, lan Ganly, MD, PhD $^{3}$, Snehal G Patel, MD ${ }^{3}$, Richard J \\ Wong, MD $^{3}$, Bhuvanesh Singh, MD, PhD $^{3}$, Jay $O$ Boyle, MD $^{3}$, Ashok R Shaha, MD $^{3}$, Jatin $\mathbf{P}$

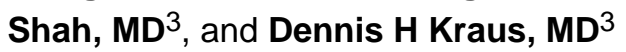 \\ ${ }^{1}$ Department of Otolaryngology-Head and Surgery, University of Toronto, Toronto, Ontario \\ ${ }^{2}$ College of Physicians and Surgeons, Columbia University, New York, NY \\ ${ }^{3}$ Head and Neck Service, Department of Surgery, Memorial Sloan-Kettering Cancer Center, New \\ York, NY
}

\begin{abstract}
Objective-The optimal type of neck dissection in head \& neck squamous cell carcinoma (SCC) with clinical cervical metastases has not been determined. The following study was performed to determine the rate of regional control with selective neck dissection (SND) in these patients.
\end{abstract}

Study Design-Case Series with planned data collection

Settings-Single Institution, cancer center

Methods\& Subject-Patients with cervical lymph node metastases from mucosal cancers of the head\& neck who were treated with SND from 2000-2010 were selected. Demographics, tumor characteristics, extent of neck dissection, adjuvant treatments, locoregional control and survival were recorded. Recurrence in the neck and disease specific survival (DSS) were primary and secondary endpoints.

Results-One hundred and eight patients underwent SND. Sixty-nine (64\%) were male. Median age was 62 (20-89). Most common primary site was oral cavity (71.3\%). Ninety-five (88\%) received adjuvant treatment. Median follow up was 21 months. Six patients (5.5\%) had isolated recurrence in the dissected neck. Patients with $\mathrm{N} 2 \mathrm{C}$ disease had poorer neck recurrence free survival. At the end of study, 64 (59.3\%) patients had no evidence of disease and 23 (21.3\%) had died of disease. Two-year DSS was $76.9 \%$. Number of positive nodes ( $\mathrm{p}=0.026)$, and positive surgical margins $(\mathrm{p}=0.001)$ among others were predictors of poorer DSS.

Conclusion-In highly selected group of patients with cervical lymph node metastases from head \& neck SCC, selective neck dissection is effective in controlling the disease in the neck, when performed in the setting of multi-modality treatment including adjuvant radiotherapy or radio-chemotherapy.

Corresponding Author: Babak Givi, MD, Microvascular-Head \& Neck Reconstruction Fellow, Department of Otolaryngology, Head and Neck Surgery, University of Toronto, 200 Elizabeth St., Rm 8N879, $8^{\text {th }}$ floor, Toronto, ON Canada M5G 2C4, Fax: 4163404727 , babak.givi@utoronto.ca.

Presented at the Annual Meeting of AAO-HNSF, September 2011, San Francisco, CA 


\section{Keywords}

Neck dissection; Head and Neck Cancer; Surgical treatment

\section{Introduction}

Neck dissection has been an integral part of the initial treatment of advanced stage head and neck cancers. Radical neck dissection, as described by Crile ${ }^{1}$, is the most comprehensive operation to remove the lymphatics of the lateral neck. However, the morbidity of this operation is quite significant in terms of shoulder dysfunction ${ }^{2}$ and venous obstruction due to removal of the internal jugular vein, in bilateral cases. Head and neck surgeons, initially, attempted to preserve the noninvolved structures. Thus the "functional neck dissection" developed by Suarez ${ }^{3}$, and popularized by Boca ${ }^{4}$, became popular in the 1970s and 1980s. After patterns of lymphatic spread of mucosal head and neck cancers were described by Lindberg ${ }^{5}$ and Shah ${ }^{6}$, the concept of selective neck dissection came into existence. In selective neck dissection, only the "at risk" lymphatic groups are removed. This concept soon became the standard of care, when the operation was done in the context of the clinically negative neck. Many studies have shown that elective, selective neck dissection is as effective as comprehensive neck dissection in controlling the disease in the neck, with decreased morbidity ${ }^{7-9}$. The next step in extending this concept was to apply selective neck dissection to the clinically positive neck, when the object of the operation is to remove known metastatic disease in the neck. The initial reports were concerning due to high rates of failure in the neck, particularly for N2/3 disease ${ }^{8}$. More recent studies have reported excellent regional control with selective neck dissection in selected group of patients with cervical nodal metastases, especially when adjuvant radiotherapy was used ${ }^{101112}$. Many unanswered questions on this topic still remain: which patients are the best candidates for less extensive operations? What factors are associated with better or worse outcome following this operation?

In the present report, we examined a group of patients with clinical cervical nodal metastases who underwent selective neck dissection. The purpose of the study was to determine the rate of regional failure, factors associated with failure in the neck, and the effect of regional failure on disease specific survival (DSS).

\section{Methods}

The study was approved by the institutional review board of Memorial Sloan-Kettering Cancer Center (MSKCC) before data collection. All patients treated at MSKCC head and neck service are entered into a prospective database. All previously untreated patients who underwent neck dissection with diagnosis of squamous cell carcinoma (SCC) from a head and neck mucosal primary site between 2000 and 2010 were retrospectively reviewed. Patients without clinical or radiographic evidence of cervical lymph node metastases preoperatively, were excluded. Patients who underwent comprehensive neck dissection, and those who did not have evidence of disease on the surgical pathology specimen were also 
excluded. The decision to perform selective neck dissection was at the discretion of the treating surgeon.

The following data were collected on the remaining patients: Demographics, primary site, stage, extent of operation, pathologic characteristics of specimen, adjuvant treatments, recurrence, disease specific survival (DSS) and overall survival. Stage of the disease was defined using the 7th edition of the American Joint Committee on Cancer (AJCC) staging manual ${ }^{13}$. Extent of surgery was based on the classification of cervical lymphatics and standard neck dissection nomenclature as defined by American Head \& Neck Society ${ }^{14}$. The number of positive nodes, extracapsular spread, and total number of nodes removed, were recorded. Presence of positive surgical margins at the primary site, perineural invasion, and lymphovascular invasion were also recorded. Recurrence was defined as local, regional and distant. Regional recurrence was further divided into recurrence in the field of dissection of dissected neck and appearance of new metastatic disease in the non-dissected, contralateral neck. Salvage treatments (if attempted) and outcomes were documented. Regional recurrence free survival was defined as the time interval between the date of operation and documentation of recurrence in the neck. Patients who had not developed recurrence in the neck were censored at the time of death, or last follow up visit whichever occurred earlier, irrespective of local and/or distant recurrence.

For DSS analysis, patients who were alive and free of disease were censored at their last follow up visit at MSKCC or at the time of death. Patients who died after their last visit at MSKCC were recorded as died of other causes if they had been free of disease within 6 months of the date of death or a specific cause of death was mentioned in the chart.

Otherwise, patients were recorded as dead of disease. Regional recurrence free survival, DSS and overall survival were calculated by the method of Kaplan-Meier. The effects of gender, age ( $>60$ versus $<60$ ), primary site, tumor stage, surgical margins, perineural invasion, lymphovascular invasion, nodal stage, number of positive nodes, extracapsular spread, adjuvant treatments, were determined by univariate analysis using log rank test. All variables were entered into a multinominal regression analysis model. A p value of less than 0.05 was considered significant. All analyses were done on SPSS, version 19 (SPSS Inc., Chicago, IL).

\section{Results}

From 2000 to 2010, three hundred and thirty patients underwent neck dissection for mucosal SCC of the head and neck. Ninety patients with elective neck dissection, and 113 with comprehensive neck dissection were excluded. One hundred and twenty-seven patients were identified with clinical nodal metastases who were treated by selective neck dissection. In this group, nineteen had prior treatments directed toward neck (radiotherapy) or systemic therapies (chemotherapy) and were further excluded. One hundred and eight patients met the inclusion criteria. Demographic details, primary site, stage, operations and pathology are described in Table 1. All but one of the patients underwent preoperative imaging in the form of CT, MRI or PET. Eleven (10.2\%) patients had evidence of bilateral disease on preoperative assessment. The majority of patients $(88 \%)$ underwent adjuvant treatment. 
Median follow up time from the date of surgery was 21 months (0-114). During the study period, $33(30.6 \%)$ patients were identified with recurrent disease at any site. Two-year recurrence free survival was $69.4 \%$. The details of recurrent disease are shown in Figure 1. Sixteen patients (14.8\%) experienced regional recurrence. All regional recurrences occurred within 15 months surgery. Of the 16 patients with regional recurrences $3(18.8 \%)$ had not received adjuvant treatments. Two-year regional recurrence free survival was $92.2 \%$. Regional recurrence occurred in the non-dissected neck in 4 (3.7\%) patients, dissected neck in 7 (6.5\%), and both dissected and non-dissected neck in $5(4.6 \%)$. Therefore, failure in the dissected neck occurred in 12 patients (11.1\%) (Table 2). In this group, eight of the patients had synchronous local recurrence. Disease was limited only to the neck in $3(2.35 \%)$ and neck and distant sites in another $3(2.35 \%)$ patients. Thus, recurrence in the dissected neck without synchronous recurrence at the primary site occurred in 6 patients $(5.5 \%)$. Ten of the 12 recurrences in the dissected neck $(83.3 \%)$ occurred from oral cavity tumors and two from larynx primary tumors. None of the oropharynx primary tumors recurred in the dissected neck. Of the 16 patients with regional recurrence, eleven (69\%) underwent salvage treatments. Four (25\%) patient received chemotherapy and radiation; three (19\%) patients chemotherapy alone; two (13\%) patient, surgery and chemotherapy; one (6\%) patient underwent surgery and one (6\%) patient was treated with radiation. Three (19\%) patients (one by surgery, one with surgery and chemotherapy and one after chemoradiation) had complete response. Four patients (25\%) had partial response and four (25\%) patients did not respond.

At the last follow up; 64 (59.3\%) patients were disease free; 6 (5.6\%) were alive with disease; 23 patients had died of disease (21.3\%), and 15 (13.9\%) had died of other causes. Two-year disease specific and overall survival were $76.9 \%$ and $64.1 \%$, respectively. Twoyear DSS for stage III disease was $80 \%$ and $75.5 \%$ for stage IV disease. Patients with regional recurrence had poorer DSS (2-year survival: $24.5 \%$ versus $86.5 \%$ ).Regional recurrence and DSS by the nodal stage and adjuvant treatments are detailed in Figure 2.

Univariate and multivariate analysis of regional recurrence in the dissected neck, and DSS are detailed in Tables 3 and 4. On univariate analysis, bilateral neck disease was significantly associated with poorer regional recurrence free survival. Oral cavity tumors showed a trend toward poorer regional recurrence free survival $(\mathrm{p}=0.06)$. Adjuvant treatment was associated with better regional recurrence free survival in multivariate analysis. Decreased DSS was associated with oral cavity tumors, primary tumors larger than T2, positive margins of resection, and lack of adjuvant treatments on univariate analysis. Multivariate analysis showed positive margins at the primary site, number of positive nodes (more than 2) and adjuvant treatments as significant factors in DSS.

\section{Discussion}

Traynor ${ }^{15}$ in 1996 presented a group of 36 patients with clinical and pathologic disease in the neck who underwent selective neck dissection. Recurrence in the neck occurred in one patient after four years of follow up. Byers ${ }^{16}$ investigated the patterns of failure after selective neck dissection. The study does not report the overall failure rate in pathologically positive neck, but the failure rate for N2B disease was 5.1\% in the dissected field and $2.2 \%$ 
in the non-dissected field. Innode positive patients who underwent supraomohyoid neck dissection, the regional failure rate was $13.4 \%$ (N1 and N2B). The rate of failure in the N1 disease was significantly reduced by the addition of adjuvant radiotherapy (35.7\% versus 5.6\%). In the Byers' study, location of primary tumor, extracapsular extension, and location of nodes in the neck were not associated with the risk of recurrence in the neck. Ambrosch ${ }^{11}$ reported a series of 254 selective neck dissections in pathologically positive nodes. More than one-third of patients were $\mathrm{pN} 1$ (34.6\%) and the remainder were $\mathrm{pN} 2$. Ten percent had bilateral disease (pN2c). Extracapsular extension was present in $27 \%$ of positive specimens. The regional recurrence rate in the dissected field was $8.3 \%$. Two patients had recurrences that potentially could have been prevented by a comprehensive neck dissection. The rate of neck recurrence at 3 years was $4.9 \%$ for pN1 disease and $12.1 \%$ for pN2. Contrary to Byers' findings, adjuvant radiation significantly reduced the rate of regional recurrence for $\mathrm{pN} 2$ disease, but made no significant difference in pN1 disease. Extracapsular extension was not a significant factor in regional recurrence.

Andersen ${ }^{10}$ reported the results of selective neck dissection in 106 clinically and pathologically positive patients. More than half were $\mathrm{pN} 1(58,54.7 \%)$ and 28 patients were pN2 (26.4\%). Thirty-six patients (34\%) had evidence of extracapsular extension. Regional recurrence occurred in six (5.7\%). The 5-year neck recurrence free survival was 92.2\%. Neck recurrence was correlated with nodal stage early in the study, but by 5 years there was no difference in the rate of recurrence. Extracapsular spread, however, was a significant factor in both neck recurrence and DSS. More recent studies show similar results. Muzaffar ${ }^{17}$ reported 3.3\% regional recurrence with selective neck dissection in 61 patients, all treated with adjuvant radiotherapy. Patel ${ }^{12}$ in 2008reported $4 \%$ failure rate in 72 patients, comparable to $14 \%$ failure rate in 160 patients with comprehensive neck dissection. Sheppard ${ }^{18}$ in 2010 reported $4.1 \%$ regional failurein 69 patients.

In the present study we focused on a group of patients who underwent selective neck dissection with therapeutic intent. All patients had evidence of disease in the neck preoperatively and in the surgical specimen. Our study design allows us to determine the outcome of selective neck dissection in patients with low volume neck disease. In addition, we are able to critically analyze the soundness of the surgeon's judgment in such cases. A high rate of regional recurrence is a source of concern to the effectiveness of selective neck dissection in the therapeutic setting. As evidenced by the data, these patients were carefully selected by the treating physicians. There were no strict selection criteria at the time of the study; but the general philosophy of our service is to offer selective neck dissection to patients with low volume disease on preoperative imaging and no clinical or radiologic evidence of extranodal extension. Out of 221 patients with neck disease during this time period, only 108 (49\%) were treated with selective neck dissection. Almost all patients underwent preoperative imaging. Most patients had N1 disease clinically and there was no evidence of involvement of nonlymphatic structures. No patient with N3 disease was treated in this fashion. Most primary tumors were in the oral cavity or oropharynx. The general philosophy of our service is to recommend definitive radiotherapy or chemoradiotherapy to most larynx, oropharynx and Hypopharynx tumors. Patients with advanced laryngeal cancers who are treated by surgery will undergo comprehensive neck dissection and thus are not included in this study. On the pathology report, $35 \%$ had advanced primary tumors, 
almost two-thirds had N2 disease, and extracapsular extension was present in more than onethird. The majority of patients were treated with adjuvant postoperative radiation. Considering the relative advanced stage of disease in our group, the rate of neck failure was very low and comparable to previous studies. When local control was achieved at the primary tumor site, only 6 failures were observed in the dissected neck (5.5\%). Our findings validate previous studies that show low recurrence rate in selective neck dissection for patients with low volume neck disease.

Similar to prior studies, all neck recurrences occurred in the first 18 months after surgery. Since isolated regional recurrences were very low in our series, we performed the regression analysis of significant factors in neck recurrence and survival including all recurrences in the dissected neck (12 events). Univariate analysis of factors in regional recurrence identified bilateral neck disease as significant (Figure 3). Site of the primary tumor also showed a strong trend toward significance (Figure 4). In our cohort of patients, only 1 patient had a recurrence in the neck from a base of tongue primary in the contralateral un-dissected neck. This patient was successfully salvaged by neck dissection.

Patients with bilateral neck disease had a significantly higher rate of regional recurrence. Even when primary site was controlled, N2C patients comprised the majority of regional recurrent cases ( 3 out of 6). Comprehensive neck dissection might be a more fitting option in this group. Even though extracapsular extension was not significant, a trend towards higher failure rate was identified $(\mathrm{p}=0.1)$. Adjuvant treatment was the only significant factor in multivariate analysis. The limited number of events (12), however, makes drawing any conclusions difficult. These findings are similar to prior reports. Ambrosch ${ }^{11}$ did not find extracapsular extension significant, but Andersen ${ }^{10}$ identified it as significant. The number of patients who did not receive adjuvant treatments is very small. However, patients who did not have adjuvant therapy showed a higher rate of regional recurrence and poorer DSS. Most patients who did not receive adjuvant treatment had N1 disease originally and there is no difference in recurrence in this group with and without adjuvant treatments. Only 6 patients with N2 disease did not undergo adjuvant treatment. Five had significant medical comorbidities and were not deemed fit for adjuvant treatment. One patient with $\mathrm{T} 1$ tonsil cancer, completely excised and no adverse features, did not undergo adjuvant treatment. This patient was disease free 9 years after treatment. The rate of regional recurrence in N2B disease is almost similar to $\mathrm{N} 1$ disease when adjuvant treatments are used, but much higher without them. Once again, the small number of patients excludes drawing any conclusions. This observation is similar to the findings by Ambrosch ${ }^{11}$. Our policy is to recommend adjuvant radiotherapy to all patients with positive nodes regardless of the nodal stage.

Analysis of DSS shows an interesting contrast between significant factors in regional recurrence and survival. Patients who experience any recurrence have poorer survival. Factors related to the primary tumor appear to be more influential in the eventual course of the disease. Location (oral cavity), extent ( $T$ stage, number of positive nodes), and adequacy of treatment (surgical margins, adjuvant treatments) all were significant predictors of DSS. These are known important prognosticating factors. Neither bilateral neck disease nor extracapsular extension were significant. It is hard to draw conclusions based on retrospective observations, but this could be due to the effective treatments for regional 
recurrence (salvage neck dissection, re-radiation). Of the five patients with isolated regional recurrence, one underwent salvage surgery and two received radiation and chemotherapy. Two were salvaged and one didn't respond to treatment.

The difference in regional recurrence between oral cavity and oropharynx tumors is worthy of attention. To our knowledge none of the prior studies have identified a difference in the rate of "regional recurrence", after standard treatments, related to the primary site. HPV associated oropharyngeal cancers in non-smoker, non-drinkers have better survival outcome with standard treatments, ${ }^{19}$ but we are not aware of any study that shows lower regional recurrence after selective neck dissection for oropharyngeal tumors in comparison to oral cavity tumors. Since we neither have data on HPV status of specimens, nor designed our study to identify differences in the rate of regional recurrence based on the primary site, we cannot draw any further conclusions.

Our study is limited by its design in implicating its observations to a paradigm shift in treatment strategies. The definitive answer as to which patients are the best candidates for selective neck dissection in clinical nodal metastases can only be answered in the settings of a randomized clinical trial. The history of surgical trials shows that performing such an undertaking is unlikely. Ours and other similar studies may provide the best available data to impact upon this difficult decision making process.

In conclusion, we present a highly selected series of patients with cervical nodal metastases from head and neck mucosal SCC that were treated by selective neck dissection. We observed a very low rate of isolated regional failure (5.5\%). Bilateral neck disease increases the risk of regional recurrence. Two-year DSS was $80 \%$ for stage III and $75.5 \%$ for stage IV disease. Oral cavity tumors, higher $\mathrm{T}$ stage and positive margins increase the risk of death from the disease. Selective neck dissection with adjuvant treatment appears to be effective in controlling the disease in the neck. Careful selection of patients and use of adjuvant treatments are important factors in the success of this approach.

\section{References}

1. Crile GW. Excision of cancer of the head and neck. JAMA. 1906; 47:1780-6.

2. Nahum AM, Mullally W, Marmor L. A syndrome resulting from radical neck dissection. Arch Otolaryngol. 1961; 74:424-8. [PubMed: 14477989]

3. Suárez O. The problem of lymphatic and distant metastases of cancer of larynx and hypopharynx. Rev Otorrinolaringol. 1963; 23:83-99.

4. Bocca E, Pignataro O. A conservation technique in radical neck dissection. Ann Otol Rhinol Laryngol. 1967; 76:975-87. [PubMed: 6074244]

5. Lindberg R. Distribution of cervical lymph node metastases from squamous cell carcinomaof the upper respiratory and digestive tracts. Cancer. 1972; 29:1446-9. [PubMed: 5031238]

6. Shah JP. Patterns of cervical lymph node metastasis from squamous carcinomas of the upper aerodigestive tract. Am J Surg. 1990; 160:405-9. [PubMed: 2221244]

7. Strong EW. Preoperative radiation and radical neck dissection. Surg Clin North Am. 1969; 49:2716. [PubMed: 5774982]

8. Medina JE, Byers RM. Supraomohyoid neck dissection: rationale, indications, and surgical technique. Head Neck. 1989; 11:111-22. [PubMed: 2722487] 
9. Results of a prospective trial on elective modified radical classical versus supraomohyoid neck dissection in the management of oral squamous carcinoma. Brazilian Head and Neck Cancer Study Group. Am J Surg. 1998; 176:422-7. [PubMed: 9874426]

10. Andersen PE, Warren F, Spiro J, et al. Results of selective neck dissection in management of the node-positive neck. Arch Otolaryngol Head Neck Surg. 2002; 128:1180-4. [PubMed: 12365890]

11. Ambrosch P, Kron M, Pradier O, et al. Efficacy of selective neck dissection: a review of 503 cases of elective and therapeutic treatment of the neck in squamous cell carcinoma of the upper aerodigestive tract. Otolaryngol Head Neck Surg. 2001; 124:180-7. [PubMed: 11226954]

12. Patel RS, Clark JR, Gao K, et al. Effectiveness of selective neck dissection in the treatment of the clinically positive neck. Head Neck. 2008; 30:1231-6. [PubMed: 18642289]

13. Edge, SB., American Joint Committee on Cancer. AJCC cancer staging manual. 7th. New York: Springer; 2010. p. xiv, 648

14. Robbins KT, Shaha AR, Medina JE, et al. Consensus statement on the classification and terminology of neck dissection. Arch Otolaryngol Head Neck Surg. 2008; 134:536-8. [PubMed: 18490577]

15. Traynor SJ, Cohen JI, Gray J, et al. Selective neck dissection and the management of the nodepositive neck. Am J Surg. 1996; 172:654-7. [PubMed: 8988670]

16. Byers RM, Clayman GL, McGill D, et al. Selective neck dissections for squamous carcinoma of the upper aerodigestive tract: patterns of regional failure. Head Neck. 1999; 21:499-505. [PubMed: 10449664]

17. Muzaffar K. Therapeutic selective neck dissection: a 25-year review. Laryngoscope. 2003; 113:1460-5. [PubMed: 12972914]

18. Shepard PM, Olson J, Harari PM, et al. Therapeutic selective neck dissection outcomes. Otolaryngol Head Neck Surg. 2010; 142:741-6. [PubMed: 20416466]

19. Ang KK, Harris J, Wheeler R, et al. Human papillomavirus and survival of patients with oropharyngeal cancer. N Engl J Med. 2010; 363:24-35. [PubMed: 20530316] 


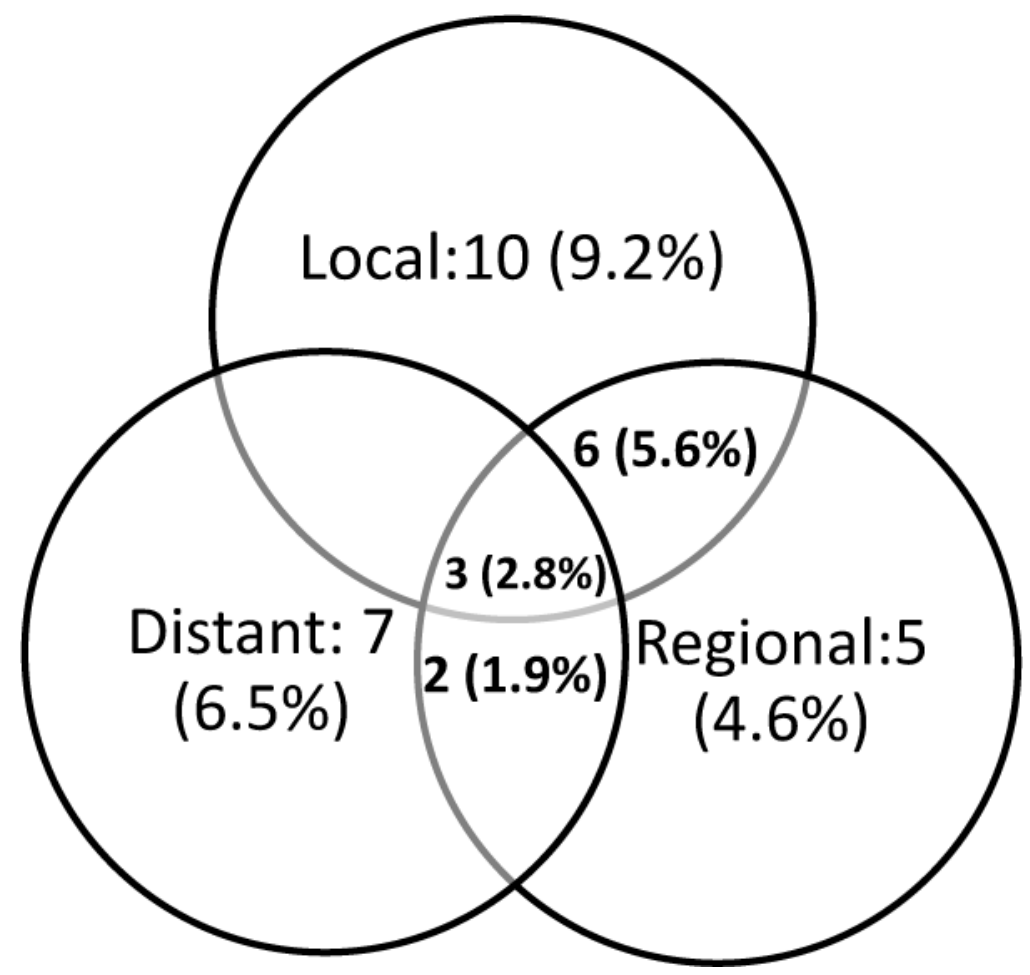

Figure 1.

Details of recurrent disease. Thirty-three (30.6\%) patients experienced recurrence, Nineteen $(17.6 \%)$ had local failure, sixteen (14.8\%) had regional failures and twelve patients developed distant metastases (11.1\%). The breakdown of the failures is illustrated in the figure. 


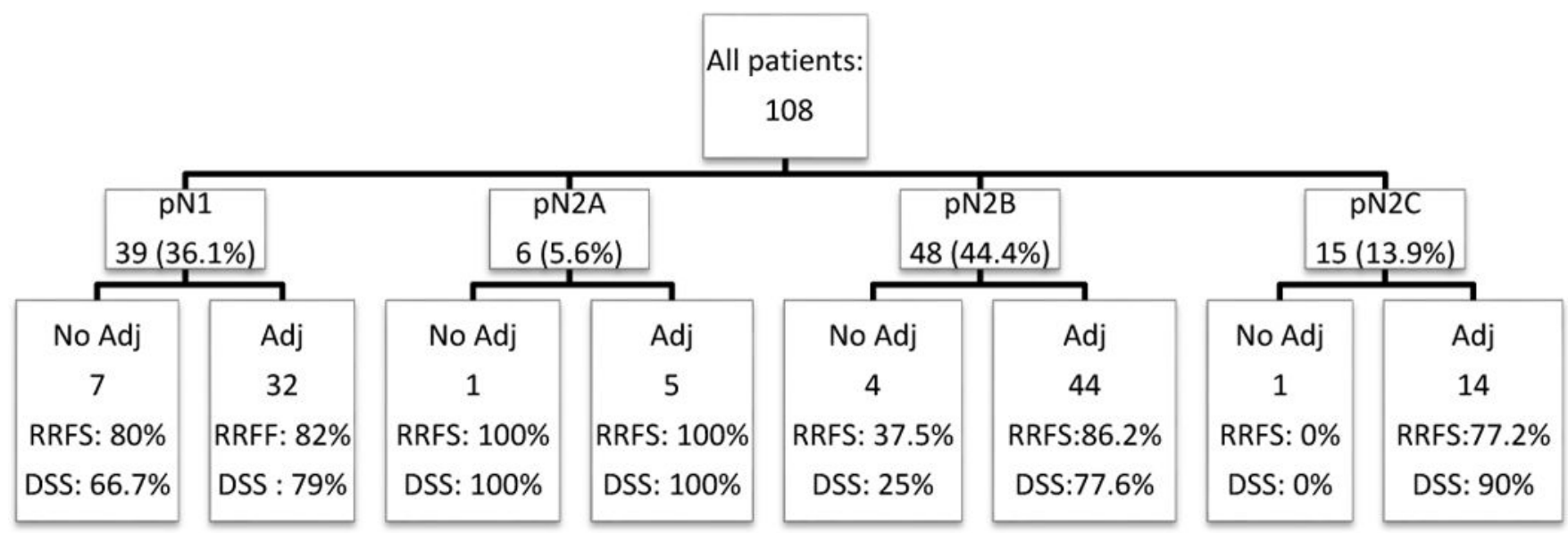

Figure 2.

Regional recurrence free survival (RRFS) and disease specific survival (DSS) for the entire cohort divided by the stage of the disease in the neck and adjuvant treatments (Adj). 


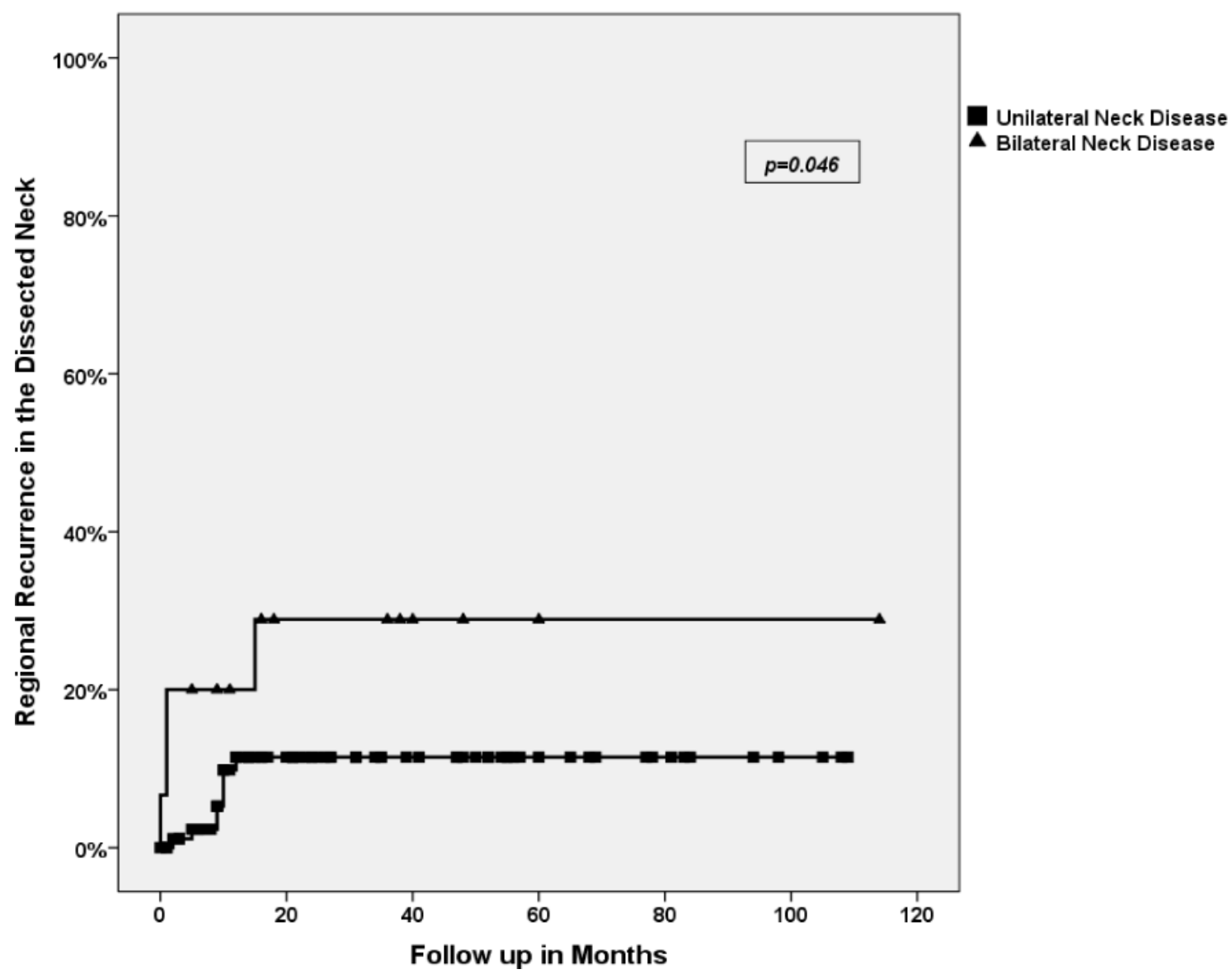

Figure 3.

Regional recurrence in the dissected neck in unilateral (N: 8/93) versus bilateral neck disease (N: 4/15). Three-year regional recurrence free survival was $88.6 \%$ for unilateral neck disease versus $71.1 \%$ for bilateral disease. 


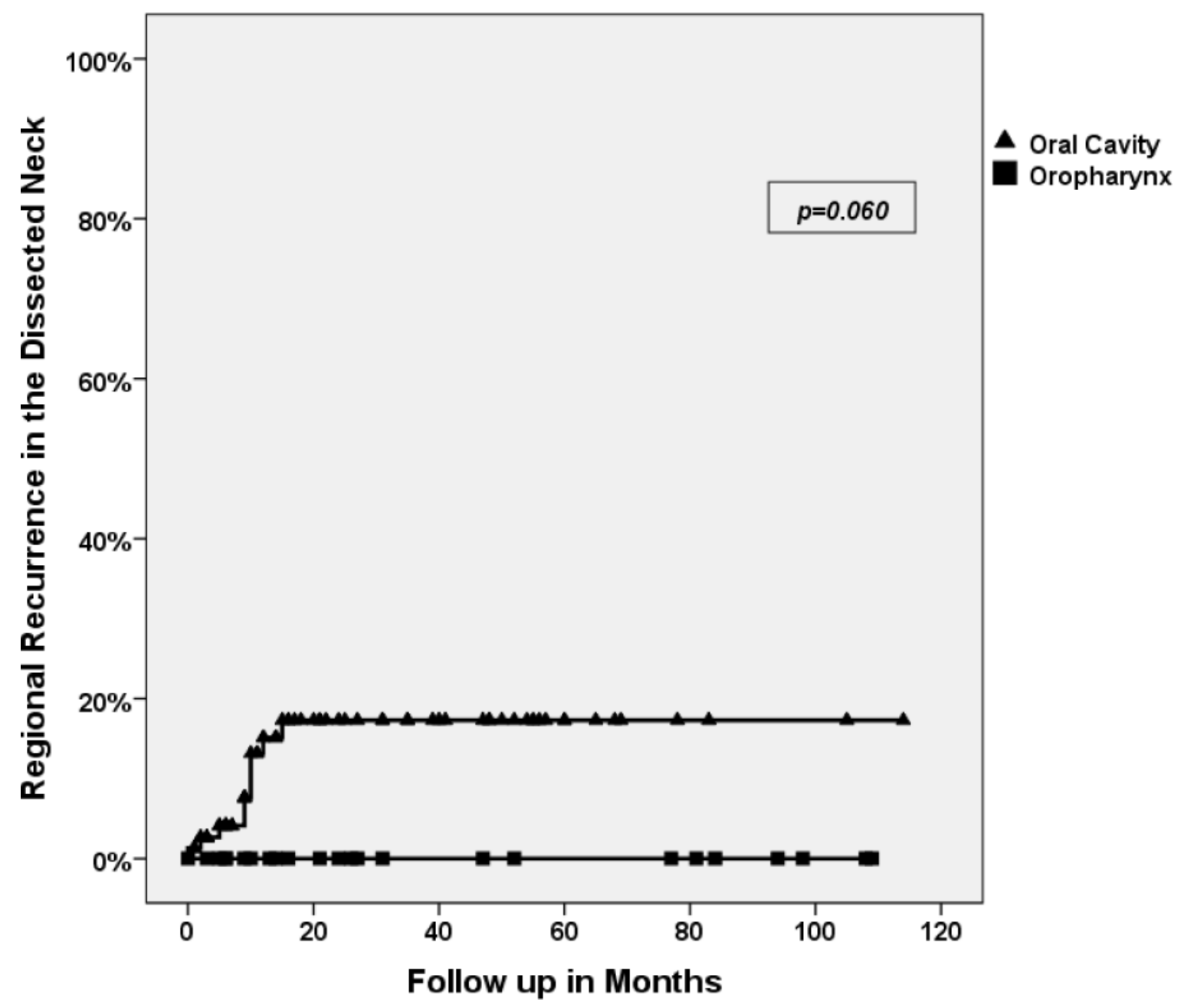

Figure 4.

Regional recurrence in the dissected neck in Oral cavity (N: 10/77) versus Oropharynx $(0 / 24)$ primary tumors. There was a strong trend for better regional control in oropharynx primaries $(\mathrm{p}=0.060)$. Two-year regional recurrence free survival was $82.7 \%$ for oral cavity tumors versus $100 \%$ for oropharyngeal tumors. 
Table 1

Demographic data and clinical stage of the tumor:

\begin{tabular}{|c|c|c|}
\hline Factor & Number (\%) & Number (\%) \\
\hline \multicolumn{3}{|l|}{ Demographics: } \\
\hline Male/Female & $69(64 \%) / 39(36 \%)$ & \\
\hline Median age at diagnosis & $62(20-89)$ & \\
\hline \multicolumn{3}{|l|}{ Primary tumor site } \\
\hline Oral cavity & $77(71.3 \%)$ & \\
\hline Oropharynx & $24(22.2 \%)$ & \\
\hline Larynx & $5(4.6 \%)$ & \\
\hline Hypopharynx & $1(0.9 \%)$ & \\
\hline Nasopharynx & $1(0.9 \%)$ & \\
\hline \multicolumn{3}{|l|}{ Staging: } \\
\hline T Stage & Clinical & Pathological \\
\hline $\mathrm{Tx}$ & $14(13 \%)$ & $10(9.3 \%)$ \\
\hline $\mathrm{T} 1$ & $16(14.8 \%)$ & $32(29.6 \%)$ \\
\hline $\mathrm{T} 2$ & $48(44.4 \%)$ & $29(26.9 \%)$ \\
\hline $\mathrm{T} 3$ & $10(9.3 \%)$ & $10(9.3 \%)$ \\
\hline $\mathrm{T} 4$ & $20(18.5 \%)$ & $27(25 \%)$ \\
\hline \multicolumn{3}{|l|}{ Nodal Stage } \\
\hline N1 & $55(50.9 \%)$ & $39(36.1 \%)$ \\
\hline $\mathrm{N} 2 \mathrm{~A}$ & $9(8.3 \%)$ & $6(5.6 \%)$ \\
\hline $\mathrm{N} 2 \mathrm{~B}$ & $33(30.6 \%)$ & $48(44.4 \%)$ \\
\hline $\mathrm{N} 2 \mathrm{C}$ & $11(10.2 \%)$ & $15(13.9 \%)$ \\
\hline \multicolumn{3}{|l|}{ Overall } \\
\hline III & & $32(29.6 \%)$ \\
\hline IVa & & $76(70.4 \%)$ \\
\hline \multicolumn{3}{|l|}{ Extent of neck dissection: } \\
\hline & Ipsilateral Neck (108) & Contralateral Neck (23) \\
\hline I-III (Supraomohyoid) & $12(11.1 \%)$ & $4(3.7 \%)$ \\
\hline I-IV (Anterolateral) & $86(79.6 \%)$ & $13(12.1 \%)$ \\
\hline II-IV (Lateral) & $5(4.6 \%)$ & $6(5.5 \%)$ \\
\hline II-V (posterolateral) & $5(4.6 \%)$ & \\
\hline \multicolumn{3}{|l|}{ Pathology details: } \\
\hline Median number of nodes removed: & $32(4-101)$ & \\
\hline Median number of positive nodes: & $2(1-19)$ & \\
\hline Extracapsular Spread: & $38(35.2 \%)$ & \\
\hline Positive surgical margins at the primary site & $12(11.1 \%)$ & \\
\hline Adjuvant Treatments: & $95(88 \%)$ & \\
\hline Radiation & $50(46.3 \%)$ & \\
\hline
\end{tabular}




\begin{tabular}{|l|c|l|}
\hline Factor & Number (\%) & Number (\%) \\
Chemoradiation & $42(38.9 \%)$ & \\
Chemotherapy & $3(2.8 \%)$ & \\
\hline
\end{tabular}


Table 2

Details of cases with recurrence in the dissected neck:

\begin{tabular}{|c|c|c|}
\hline Factor & Number (\%) & Number $(\%)$ \\
\hline & $\begin{array}{l}\text { All dissected neck failures } \\
\mathrm{N}=12\end{array}$ & $\begin{array}{l}\text { Dissected neck failure without local failure } \\
\mathrm{N}=6\end{array}$ \\
\hline Male/Female & $7 / 5(58.3 \% / 41.7 \%)$ & $4 / 2(67 \% / 33 \%)$ \\
\hline Median age & 64.5 & 68 \\
\hline \multicolumn{3}{|l|}{ Primary Site: } \\
\hline Oral Cavity & $10(83.3 \%)$ & $4(67 \%)$ \\
\hline Larynx & $2(16.7 \%)$ & $2(33 \%)$ \\
\hline \multicolumn{3}{|l|}{ Type of Neck dissection: } \\
\hline I-III (supraomohyoid) & $1(8.3 \%)$ & 0 \\
\hline I-IV (Anterolateral) & $9(75 \%)$ & $4(66.6 \%)$ \\
\hline II-IV (Lateral) & $1(8.3 \%)$ & $1(16.7 \%)$ \\
\hline II-V (Posterolateral) & $1(8.3 \%)$ & $1(16.7 \%)$ \\
\hline Bilateral Neck Dissection: & $7(58.3 \%)$ & $4(66.6 \%)$ \\
\hline \multicolumn{3}{|l|}{$\mathrm{T}$ Stage of the primary } \\
\hline $\mathrm{T} 1$ & $3(25 \%)$ & $1(16.7 \%)$ \\
\hline $\mathrm{T} 2$ & $4(33.3 \%)$ & $1(16.7 \%)$ \\
\hline $\mathrm{T} 3$ & $1(8.3 \%)$ & $1(16 / 7 \%)$ \\
\hline $\mathrm{T} 4 \mathrm{a}$ & $4(33.3 \%)$ & $3(50 \%)$ \\
\hline \multicolumn{3}{|l|}{ Pathologic N stage } \\
\hline $\mathrm{N} 1$ & $6(50 \%)$ & $2(33.3 \%)$ \\
\hline $\mathrm{N} 2 \mathrm{~B}$ & $2(16.7 \%)$ & $2(16.7 \%)$ \\
\hline $\mathrm{N} 2 \mathrm{C}$ & $4(33.3 \%)$ & $3(50 \%)$ \\
\hline Median number of positive nodes & 1.5 & 3.5 \\
\hline Positive surgical margins at the primary site & $2(16.7 \%)$ & $1(16.7 \%)$ \\
\hline Extracapsular Extension & $6(50 \%)$ & $4(67 \%)$ \\
\hline Perineural invasion & $6(50 \%)$ & $1(16.7 \%)$ \\
\hline Lymphovascular invasion & $3(25 \%)$ & $1(16.7 \%)$ \\
\hline Adjuvant treatment & $9(75 \%)$ & $3(50 \%)$ \\
\hline
\end{tabular}


Table 3

Bivariate analysis of regional recurrence free survival (RRFS) and disease specific survival (DSS):

\begin{tabular}{|c|c|c|c|c|}
\hline \multirow[b]{2}{*}{ Factor $(\mathbf{n})$} & \multicolumn{2}{|c|}{ Regional Recurrence Free Survival (RRFS) } & \multicolumn{2}{|c|}{ Disease Specific Survival (DSS) } \\
\hline & $\begin{array}{l}\text { 2-Yr } \\
\text { RRFS }\end{array}$ & p value & $\begin{array}{l}\text { 2-Yr } \\
\text { DSS }\end{array}$ & p value \\
\hline Gender & & 0.767 & & 0.233 \\
\hline Male (69) & $87.2 \%$ & & $73.3 \%$ & \\
\hline Female (39) & $83.9 \%$ & & $83.4 \%$ & \\
\hline Age & & 0.777 & & 0.227 \\
\hline$<60(49)$ & $87.3 \%$ & & $79.5 \%$ & \\
\hline$>60(59)$ & $84.6 \%$ & & $74.7 \%$ & \\
\hline Primary site & & 0.060 & & 0.004 \\
\hline Oral cavity (77) & $82.7 \%$ & & $69.2 \%$ & \\
\hline Oropharynx (24) & $100 \%$ & & $100 \%$ & \\
\hline $\begin{array}{l}\text { Primary } \mathrm{T} \\
\text { Stage }\end{array}$ & & 0.311 & & 0.003 \\
\hline$<\mathrm{T} 3(71)$ & $87.2 \%$ & & $85.4 \%$ & \\
\hline$\geq \mathrm{T} 3(37)$ & $85.9 \%$ & & $60.3 \%$ & \\
\hline Bilateral Neck Disease & & 0.046 & & 0.921 \\
\hline No (93) & $88.6 \%$ & & $76.1 \%$ & \\
\hline Yes (15) & $77.1 \%$ & & $83.1 \%$ & \\
\hline Number of Positive nodes: & & 0.675 & & 0.164 \\
\hline $1-2(72)$ & $84 \%$ & & $83 \%$ & \\
\hline$>2(36)$ & $91.7 \%$ & & $83 \%$ & \\
\hline Extracapsular extension & & 0.192 & & 0.653 \\
\hline No (70) & $87.9 \%$ & & $79.8 \%$ & \\
\hline Yes (38) & $82.9 \%$ & & $70 \%$ & \\
\hline Margins on primary site & & 0.266 & & 0.000 \\
\hline Negative (96) & $87.1 \%$ & & $82.8 \%$ & \\
\hline Positive (12) & $78.6 \%$ & & $30.6 \%$ & \\
\hline Perineural Invasion & & 0.516 & & 0.126 \\
\hline No (65) & $88.8 \%$ & & $82.5 \%$ & \\
\hline Yes (43) & $82.1 \%$ & & $69.7 \%$ & \\
\hline Lymphovascular Invasion & & 0.409 & & 0.407 \\
\hline No (70) & $83.5 \%$ & & $74.3 \%$ & \\
\hline Yes (38) & $90.5 \%$ & & $80.8 \%$ & \\
\hline Adjuvant Treatments & & 0.051 & & 0.003 \\
\hline
\end{tabular}

Otolaryngol Head Neck Surg. Author manuscript; available in PMC 2018 January 29. 


\begin{tabular}{|l|l|l|l|l|}
\hline & \multicolumn{2}{|l|}{ Regional Recurrence Free Survival (RRFS) } & Disease Specific Survival (DSS) \\
\hline Factor (n) & $\mathbf{2 - Y r}$ & p value & $\mathbf{2 - Y r}$ & p value \\
& RRFS & & DSS & \\
Yes (95) & $88.1 \%$ & $80.8 \%$ & \\
No (13) & $80.8 \%$ & & $46.9 \%$ & \\
\hline
\end{tabular}




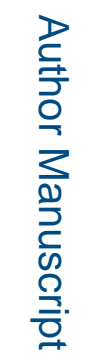

\begin{tabular}{|c|c|c|c|c|c|c|c|c|c|c|c|}
\hline 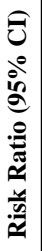 & 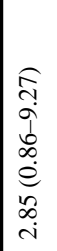 & 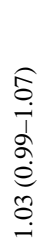 & 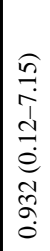 & 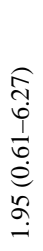 & 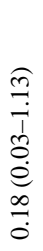 & 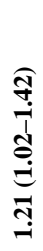 & 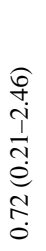 & 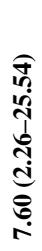 & 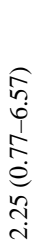 & 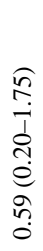 & 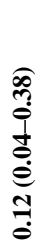 \\
\hline 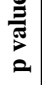 & $\mid \begin{array}{l}\mathscr{O} \\
\stackrel{\leftrightarrow}{0} \\
\stackrel{0}{0}\end{array}$ & $\begin{array}{l}\text { तิ่ } \\
\text { है }\end{array}$ & $\stackrel{n}{a}$ & $\begin{array}{l}\hat{\delta} \\
\text { ஸे }\end{array}$ & $\begin{array}{l}\text { : } \\
:\end{array}$ & 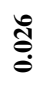 & $\begin{array}{l}\text { ô } \\
0 \\
0\end{array}$ & $\stackrel{\bar{\Xi}}{\stackrel{0}{0}}$ & 9 & 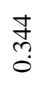 & 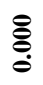 \\
\hline
\end{tabular}

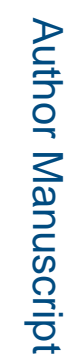

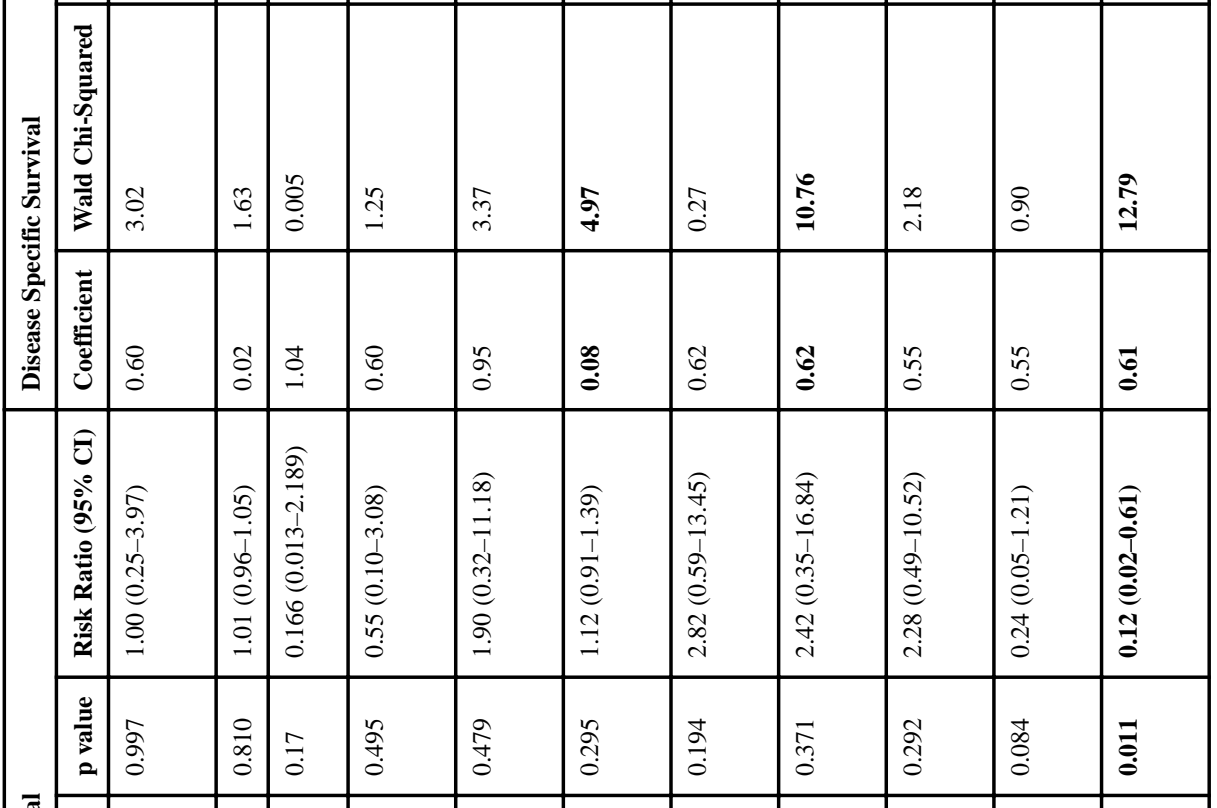

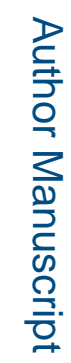

\begin{tabular}{|c|c|c|c|c|c|c|c|c|c|c|c|c|}
\hline 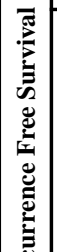 & & $\stackrel{8}{8}$ & $\mid \begin{array}{l}\infty \\
\stackrel{\infty}{0} \\
0 \\
0\end{array}$ & $\stackrel{\infty}{\rightarrow}$ & \begin{tabular}{|l}
$\infty$ \\
$\infty$ \\
$\infty$ \\
$\infty$ \\
0
\end{tabular} & $\begin{array}{l}8 \\
\text { nn. } \\
0\end{array}$ & 兽 & $\stackrel{\rho}{\circ}$ & $\mid \begin{array}{l}0 \\
\infty \\
0\end{array}$ & $\exists$ & $\begin{array}{l}\hat{i} \\
\text { in }\end{array}$ & $\overline{5}$ \\
\hline 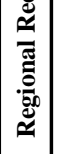 & 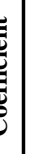 & ?̊. & $\mid \begin{array}{c}0 \\
0 \\
0\end{array}$ & 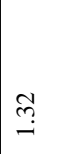 & $\begin{array}{l}\infty \\
\infty \\
0 \\
0\end{array}$ & $\bar{a}$ & $\overline{0}$ & $\begin{array}{l}\infty \\
\infty \\
\infty\end{array}$ & oे & $\stackrel{\substack{\infty \\
0}}{\stackrel{0}{0}}$ & $\mid \begin{array}{l}+ \\
\infty \\
0\end{array}$ & 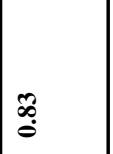 \\
\hline & E & 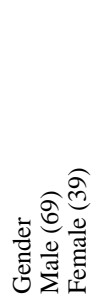 & $\begin{array}{l}8 \\
< \\
\end{array}$ & 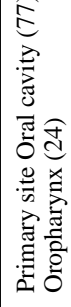 & 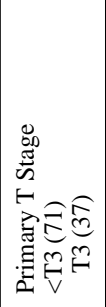 & 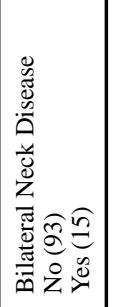 & 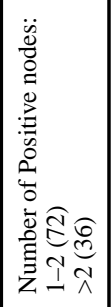 & 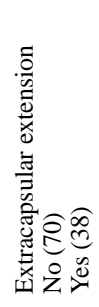 & 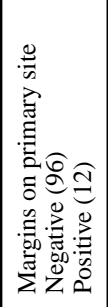 & 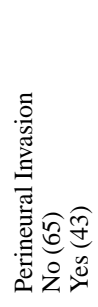 & 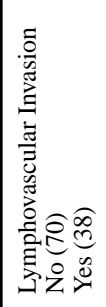 & 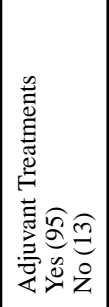 \\
\hline
\end{tabular}

Otolaryngol Head Neck Surg. Author manuscript; available in PMC 2018 January 29. 